\title{
Nonparametric confidence intervals for tail dependence based on copulas
}

\author{
Cheikh Tidiane Seck ${ }^{1,2, *}$, Diam Ba ${ }^{1}$ and Gane Samb Lo ${ }^{1,3,4}$ \\ ${ }^{1}$ LERSTAD, Université Gaston Berger, Sénégal \\ ${ }^{2}$ Université Alioune Diop de Bambey, Sénégal \\ ${ }^{3}$ LSTA, Université Pierre et Marie Curie, France \\ ${ }^{4}$ AUST - African University of Sciences and Technology, Abuja, Nigeria
}

Received November 20, 2016; Accepted December 05, 2016

Copyright (c) 2016, Afrika Statistika and Statistics and Probability African Society (SPAS). All rights reserved

\begin{abstract}
We propose nonparametric asymptotic confidence intervals for the upper and lower tail dependence coefficients. These latter are obtained from confidence bands established for the copula function itself and based upon three kernel-type estimators. We show the performance of these confidence intervals through a simulation study. We also apply these results to financial data stemming from the CAC 40 stock index which reveals the existence of extreme dependence between larger values of the opening and closing prices for this index during the considered period.
\end{abstract}

Résumé. Ce papier présente des intervalles de confiance asymptotiques pour les coefficients de dépendance de queue inférieure et supérieure. Ces derniers sont obtenus de façon nonparamétrique à partir de bandes de confiance presque sûres obtenues pour la fonction copule elle même et basées sur des estimateurs à noyau. Nous montrons ensuite la performance de ces intervalles de confiance à travers une étude de simulation. Une application de ces résultats sur des données financières issues de l'indice boursier CAC 40 révèlent une dépendance extrême entre les valeurs d'ouverture et de fermeture de cet indice durant la période étudiée.

Key words: Tail dependence coefficient, Confidence intervals, Kernel estimators, Copula function.

AMS 2010 Mathematics Subject Classification : 62G20, 62H20, 62P05.

${ }^{*}$ Corresponding author Cheikh Tidiane Seck : cheikhtidiane.seck@uadb.edu.sn

Diam ba :diamba79@gmail.com

Gane Samb Lo :ganesamblo@ganesamblo.net 


\section{Introduction}

Copula is a mathematical tool which handles the dependence structure between two or several random variables. It gives a more complete characterization of dependence than the classical measures such as the linear correlation coefficient, Kendall's tau and Spearman's rho. Formally, a $d$-copula, $d \geq 1$ integer, is a function $C:[0,1]^{d} \rightarrow[0,1]$ which is invariant to strictly increasing transformations of the components. But it can also be seen as a multivariate distribution function with uniform margins. That is, for $d=2$ and $0 \leq u, v \leq 1$,

$$
C(u, v)=\mathbb{P}(U \leq u, V \leq v), \quad U, V \sim \mathcal{U}(0,1)
$$

Copula is a useful tool which can help to express many other association measures as shown below, for example, in case of the tail dependence coefficient.

Let $(X, Y)$ be a couple of random variables defined on a probability space $(\Omega, \mathcal{A}, \mathbb{P})$ with marginal distributions $F$ and $G$. Let $F^{-1}(u)=\inf \{x: F(x) \geq u\}$ and $G^{-1}(v)=\inf \{y$ : $G(y) \geq v\}, 0 \leq u, v \leq 1$ denote the quantile functions associated with $F$ and $G$, respectively. The tail dependence measure of the joint distribution function of $(X, Y)$ is characterized by two coefficients : the upper tail dependence coefficient

$$
\lambda_{U}=\lim _{u \rightarrow 1^{-}} \mathbb{P}\left(X>F^{-1}(u) / Y>G^{-1}(u)\right)
$$

and the lower tail dependence coefficient

$$
\lambda_{L}=\lim _{u \rightarrow 0^{+}} \mathbb{P}\left(X<F^{-1}(u) / Y<G^{-1}(u)\right) .
$$

These two coefficients may be re-written (see e.g., Joe, 1997) in terms of the copula function $C(u, v)$. Precisely, one has

$$
\lambda_{U}=\lim _{u \rightarrow 1^{-}} \frac{1-2 u+C(u, u)}{1-u} \quad \text { and } \quad \lambda_{L}=\lim _{u \rightarrow 0^{+}} \frac{C(u, u)}{u} .
$$

The concept of tail dependence may be understood as the conditional probability of observing an extreme value for one variable given that the other being in an extreme state. One can remark that the tail dependence coefficient, say $\lambda$, has the copula property which is invariant under strictly increasing transformations of marginals. For $\lambda=0$, the extremes are asymptotically independent ; for $\lambda=1$ the extremes are perfectly correlated and for $0<$ $\lambda<1$, the extremes are asymptotically dependent. Note that the concept of tail dependence can also be generalized in a multivariate framework (see, for instance Joe, 1997).

The tail dependence coefficient (TDC) is applied in many fields for evaluating the probability of simultaneous occurence of extremes events.

For instance, in Insurance when we are concerned with concomittant sinistrality in two branches of insurance. Given that a sinistre happens in one branch, one can employ the TDC coefficient to determine the probability that another sinistre of same intensity occurs on the other branch.

In Finance, when studying simultaneous losses of two assets, we may also apply the TDC which measures the probability that one asset loses an amount greater than a quantile of order $u$, given the other asset has already lost an amount greater than the same $u$-order quantile of its distribution.

Journal home page: www.jafristat.net ; www.projecteuclid.org/as 
The TDC may be interpreted in terms of Value-at-Risk (VaR), as the quantiles $F^{-1}(u)$ and $G^{-1}(u)$ represent the $\mathrm{VaR}$ if their loss distributions are assumed to be positive. In this case the TDC provides the probability that the two variables exceed their VaR at threshold $u$, as $u \rightarrow 1$.

In Economy, inflation periods are often characterized by simultaneous increasing of different product prices. Then, one can apply the TDC coefficient to estimate extremal dependence between the prices of these products.

There are three main approaches to the estimation of tail dependence coefficients. The first is parametric and is based on the hypothesis that the dependence structure of the variables is represented by an Archimedian copula, combined with the result of Juri and Wuthrich (2002) on this family of copula.

The second approach is based upon a factor model connecting the two variables by means of a linear model with a noise. It is semiparametric (see, Malevergne and Sornette, 2004).

The third approach is nonparametric and has gained much importance. Making use of the empirical copula function and a property of the logarithm function, Coles et al. (1999) proposed an estimator for the upper tail dependence coefficient $\lambda_{U}$. By using the notion of tail copula, Schmidt and Stadtmuller (2006) proposed estimators for both the upper and lower tail coefficients $\lambda_{U}$ and $\lambda_{L}$. Another nonparametric approach to estimate tail dependence is that of Poon et al. (2004), who transformed the initial variables to Fréchet variables according to Ledford and Tawn (1998).

Our aim in this paper is to contribute to this literature by providing asymptotic optimal confidence intervals for the upper and lower tail dependence coefficients $\lambda_{U}$ and $\lambda_{L}$. These latter are obtained from confidence bands derived for the copula curve $C(u, v)$ itself. We shall deal with kernel estimators introduced in Chen and Huang (2007), Gijbels and Mielniczuk (1990) and Fermanian et al. (2004), respectively called the local linear, the mirror-reflection and the transformation estimators and defined in the next section.

The paper is organized as follows. In the next section, we establish asymptotic nonparametric confidence intervals for $\lambda_{U}$ and $\lambda_{L}$. Section 3 presents a simulation study with two Archimedian copula families and then an application to test for extremal dependence between financial data sets. The paper is ended by an Appendix, where some proofs are displayed.

\section{Nonparametric confidence intervals}

Let $\left(X_{1}, Y_{1}\right), \cdots,\left(X_{n}, Y_{n}\right)$ be an independent and identically distributed sample of the random vector $(X, Y)$ with joint distribution function $H$ and marginals distributions $F$ and $G$. Denote by $H_{n}, F_{n}$ and $G_{n}$ the empirical distribution functions of $H, F$ and $G$, respectively. Nonparametric estimation of copulas has been largely investigated in the literature. In order to test for independence, Deheuvels (1979) proposed the following estimator

$$
\hat{C}_{n}(u, v)=\frac{1}{n} \sum_{i=1}^{n} \mathbb{I}\left\{\hat{U}_{i} \leq u, \hat{V}_{i} \leq v\right\}, \quad \text { with } \hat{U}_{i}=F_{n}\left(X_{i}\right), \hat{V}_{i}=G_{n}\left(Y_{i}\right),
$$

which is asymptotically equivalent (up to a term $O\left(n^{-1}\right)$ ) to the estimator directly based on the Sklar's Theorem given by

$$
C_{n}(u, v)=H_{n}\left(F_{n}^{-1}(u), G_{n}^{-1}(v)\right),
$$

Journal home page: www.jafristat.net ; www.projecteuclid.org/as 
where $F_{n}^{-1}, G_{n}^{-1}$ are the empirical quantile functions for $F_{n}$ and $G_{n}$.

Kernel smoothed versions estimators have also been introduced but they suffer from a boundary bias due to the fact that a copula has its support on the compact set $[0,1]^{2}$. To overcome the problem of boundary bias, Chen and Huang (2007) proposed a local linear kernel estimator and a simple mathematical correction that removes the bias at the corner $(1,1)$. Their estimator is defined into two steps. First, they estimate the marginal distributions $F$ and $G$, respectively, by

$$
\hat{F}_{n}(x)=\frac{1}{n} \sum_{i=1}^{n} K\left(\frac{x-X_{i}}{b_{1 n}}\right) \quad \text { and } \quad \hat{G}_{n}(y)=\frac{1}{n} \sum_{i=1}^{n} K\left(\frac{y-Y_{i}}{b_{2 n}}\right),
$$

where $K(x)=\int_{-\infty}^{x} k(t) d t$, with $k(\cdot)$ being a bounded symmetric kernel function supported on $[-1,1] ; b_{1 n}$ and $b_{2 n}$ are some smoothing parameters. Second, the pseudo-observations $\hat{U}_{i}=\hat{F}_{n}\left(X_{i}\right)$ and $\hat{V}_{i}=\hat{G}_{n}\left(Y_{i}\right)$ are then used to estimate the copula $C$ which gives the joint distribution of the unobserved random variables $F\left(X_{i}\right)$ and $G\left(Y_{i}\right)$. Considering a local linear version of the kernel $k(\cdot)$ given by

$$
k_{u, h_{n}}(t)=\frac{k(t)\left\{a_{2}\left(u, h_{n}\right)-a_{1}\left(u, h_{n}\right) t\right\}}{a_{0}\left(u, h_{n}\right) a_{2}\left(u, h_{n}\right)-a_{1}^{2}\left(u, h_{n}\right)} \mathbb{I}\left\{\frac{u-1}{h_{n}}<t<\frac{u}{h_{n}}\right\},
$$

where $a_{j}\left(u, h_{n}\right)=\int_{(u-1) / h_{n}}^{u / h} t^{j} k(t) d t, \quad j=0,1,2,0<h_{n}<1$ and $u \in[0,1]$, Chen and Huang (2007) define their estimator as follows :

$$
\hat{C}_{n}^{(L L)}(u, v)=\frac{1}{n} \sum_{i=1}^{n} K_{u, h_{n}}\left(\frac{u-\hat{U}_{i}}{h_{n}}\right) K_{v, h_{n}}\left(\frac{v-\hat{V}_{i}}{h_{n}}\right)
$$

where $K_{u, h_{n}}(x)=\int_{-\infty}^{x} k_{u, h_{n}}(t) d t$ and $\hat{U}_{i}=\hat{F}_{n}\left(X_{i}\right), \quad \hat{V}_{i}=\hat{G}_{n}\left(Y_{i}\right)$

In order to remove the boundary bias, Gijbels and Mielniczuk (1990) proposed another method which generates eight copies of the original observations by reflecting them with respect to the four edges and four corners of the unit square $[0,1]^{2}$. Their proposal called the mirror-reflection estimator can be defined as

$\hat{C}_{n}^{(M R)}(u, v)=\frac{1}{n} \sum_{i=1}^{n} \sum_{l=1}^{9}\left[K\left(\frac{u-\hat{U}_{i}^{(l)}}{h_{n}}\right)-K\left(\frac{-\hat{U}_{i}^{(l)}}{h_{n}}\right)\right] \times\left[K\left(\frac{v-\hat{V}_{i}^{(l)}}{h_{n}}\right)-K\left(\frac{-\hat{V}_{i}^{(l)}}{h_{n}}\right)\right]$,

where

$$
\begin{gathered}
\left\{\left(\hat{U}_{i}^{(l)}, \hat{V}_{i}^{(l)}\right), i=1, \ldots, n, l=1, \ldots, 9\right\}= \\
\left\{\left( \pm \hat{U}_{i}, \pm \hat{V}_{i}\right),\left( \pm \hat{U}_{i}, 2-\hat{V}_{i}\right),\left(2-\hat{U}_{i}, \pm \hat{V}_{i}\right),\left(2-\hat{U}_{i}, 2-\hat{V}_{i}\right), i=1, \ldots, n\right\}
\end{gathered}
$$

To take care of the boundary bias, Omelka et al. (2009) introduced improved versions of the estimators (4) and (5) by shrinking the bandwidth $h_{n}$ near the corners of the unit square. They also proposed a modification of the estimator introduced in Fermanian et al. (2004)

Journal home page: www.jafristat.net ; www.projecteuclid.org/as 
via a transformation $\phi$ (which may be a distribution function) satisfying certain conditions. The formula they obtained, named the transformation estimator, is defined as

$$
\hat{C}_{n}^{(T)}(u, v)=\frac{1}{n} \sum_{i=1}^{n} K\left(\frac{\phi^{-1}(u)-\phi^{-1}\left(\hat{U}_{i}^{(E)}\right)}{h_{n}}\right) K\left(\frac{\phi^{-1}(v)-\phi^{-1}\left(\hat{V}_{i}^{(E)}\right)}{h_{n}}\right),
$$

where $\hat{U}_{i}^{(E)}=\frac{n}{n+1} F_{n}\left(X_{i}\right)$ and $\hat{V}_{i}^{(E)}=\frac{n}{n+1} G_{n}\left(Y_{i}\right)$.

Since the behavior of kernel-type estimators depends crucially on the bandwidth and that the selection of the optimal bandwidth is problematic, we reconsider in this paper the latter three estimators (4), (5) and (6) by letting the bandwidth $h$ being a random variable which may depend either on the observations or the location point $(u, v)$. In the sequel, we denote $h=\hat{h}_{n}(u, v)$ for all fixed $(u, v) \in[0,1]^{2}$.

To simplify notation, let $\hat{C}_{n, h}^{(.)}$represent each other of the above three estimators (4), $(5)$ and $(6)$, where (.) designs $(L L),(M R)$ or $(T)$. Our methodology for constructing confidence bands for the copula function $C$ is based on the classical decomposition bias-variance of the difference $\hat{C}_{n, h}^{(.)}-C$ into two terms. By means of the modern empirical process theory, we establish that when suitably normalized the first term, say the deviation $\hat{C}_{n, h}^{(.)}-\mathbb{E} \hat{C}_{n, h}^{(.)}$, converges uniformly in probability to a positive constant $A(c)$, as $n \rightarrow \infty$. Next, under smoothness assumptions on the copula $C$ and regularity conditions on the bandwidth $h$, we prove that as $n \rightarrow \infty$ the second term, the so-called bias $\mathbb{E} \hat{C}_{n, h}^{(.)}-C$, converges uniformly in probability to 0 , over $(u, v) \in[0,1]^{2}$, with the same rate. The combination of these two facts allows us to define asymptotic optimal simultaneous confidence bands for the copula curve $C(u, v)$, over $(u, v) \in[0,1]^{2}$, from wich we derive asymptotic confidence bounds for the upper and lower tail dependence coefficients.

Let $\left(h_{n}\right)_{n \geq 1}$ be a sequence of positive constants converging to 0 , as $n \rightarrow \infty$, and $b:[0,1]^{2} \mapsto$ $[0,1]$ a real-valued function defined, for any given parameter $\alpha>0$, as

$$
b(u, v)=\max \left\{\min \left\{u^{\alpha},(1-u)^{\alpha}\right\}, \min \left\{v^{\alpha},(1-v)^{\alpha}\right\}\right\},(u, v) \in[0,1]^{2} .
$$

Let $\left(b_{n}\right)_{n \geq 1}$ be a sequence of positive constants satisfying :

$$
b_{n} \rightarrow 0, \quad(\log n)^{-1}<b_{n}<1, \quad \sqrt{n} b_{n}^{2}=o(\sqrt{\log \log n / n}) .
$$

To establish our results, we need the following assumptions on the bandwidth $h=\hat{h}_{n}(u, v)$ : $\mathrm{H} 1)$

$$
\forall \epsilon>0, \mathbb{P}\left(\sup _{(u, v) \in[0,1]^{2}}\left|\frac{\hat{h}_{n}(u, v)}{h_{n}}-b(u, v)\right|>\epsilon\right) \longrightarrow 0, n \rightarrow \infty,
$$

H2) For some $c>0$,

$$
\mathbb{P}\left(\frac{c \log n}{n} \leq \hat{h}_{n}(u, v) \leq b_{n}, \forall 0 \leq u, v \leq 1,\right) \longrightarrow 1, n \rightarrow \infty .
$$

Now, we are ready to state our results which are set up in terms of the quantity $\hat{C}_{n, \hat{h}_{n}(u, v)}^{(\cdot)}(u, v)$, representing each of the above three estimators by substituting $(\cdot)$ for $(L L),(M R)$ and $(T)$, respectively.

Journal home page: www.jafristat.net ; www.projecteuclid.org/as 
Theorem 1. Suppose that the copula function $C$ admits bounded second-order partial derivatives on $[0,1]^{2}$ and that assumptions H.1) and H.2) hold. Then for any $\epsilon>0$, one has, as $n \rightarrow \infty$,

$$
\mathbb{P}\left(C(u, v) \in\left[\hat{C}_{n, \hat{h}_{n}(u, v)}^{(\cdot)}(u, v)-E_{n}(\epsilon), \hat{C}_{n, \hat{h}_{n}(u, v)}^{(\cdot)}(u, v)+E_{n}(\epsilon)\right], \forall 0 \leq u, v \leq 1\right) \longrightarrow 1
$$

and

$$
\mathbb{P}\left(C(u, v) \in\left[\hat{C}_{n, \hat{h}_{n}(u, v)}^{(\cdot)}(u, v)-\Delta_{n}(\epsilon), \hat{C}_{n, \hat{h}_{n}(u, v)}^{(\cdot)}(u, v)+\Delta_{n}(\epsilon)\right], \forall 0 \leq u, v \leq 1\right) \longrightarrow 0
$$

where $E_{n}(\epsilon)=(1+\epsilon) \frac{3}{R_{n}}, \quad \Delta_{n}(\epsilon)=(1-\epsilon) \frac{3}{R_{n}}$, and $R_{n}=\left(\frac{n}{2 \log \log n}\right)^{1 / 2}$.

\section{Remark.}

1) Assumption H.1) helps us to control the limiting behavior of the random bandwidth $\hat{h}_{n}(u, v)$ with a sequence of deterministic constants $h_{n} \searrow 0$. The $[0,1]$-valued function $b(u, v)$ allows to adjust down the value of bandwidth and to keep it close to zero near the corners of the unit square. As our concern is in tail dependence, this enables us to calculate the estimators near the corners $(0,0)$ and $(1,1)$ for evaluating the coefficients $\lambda_{U}$ and $\lambda_{L}$.

2) Assumption H.2) is necessary for applying Mason and Swanepoel's (2010) result, from which we derive a uniform in bandwidth LIL (law of the iterated logarithm) for the deviations of the estimators.

\section{Proof.}

The proof of the Theorem 1 is obtained by combining the results stated in the two following propositions. Their proofs are postponed to the Appendix. We also refer to Ba et al. (2015), for details.

Proposition 1. For any sequence of positive constants $\left(b_{n}\right)_{n \geq 1}$ satisfying $0<b_{n}<1, b_{n} \rightarrow$ $0, b_{n} \geq(\log n)^{-1}$, and for some $c>0$, we have for all $0<\varepsilon<1$ and all large $n$,

$$
\mathbb{P}\left\{\left|R_{n} \sup _{\frac{c \log n}{n} \leq h \leq b_{n}(u, v) \in[0,1]^{2}} \sup _{n, h}\right| \hat{C}^{(.)}(u, v)-\mathbb{E} \hat{C}_{n, h}^{(.)}(u, v)|-A(c)|>\varepsilon\right\}=o(1),
$$

where $A(c)$ is a positive constant such that $0<A(c) \leq 3$ and $R_{n}=\left(\frac{n}{2 \log \log n}\right)^{1 / 2}$.

Proposition 2. Suppose that the copula function $C(u, v)$ admits bounded second-order partial derivatives on $[0,1]^{2}$. Then for any sequence of positive constants $\left(b_{n}\right)_{n \geq 1}$ satisfying $0<b_{n}<1, \sqrt{n} b_{n}^{2} / \sqrt{\log \log n}=o(1)$, and for some $c>0$, we have for all $0<\varepsilon<1$ and all large $n$,

$$
\mathbb{P}\left\{R_{n} \sup _{\frac{c \log n}{n} \leq h \leq b_{n}} \sup _{(u, v) \in[0,1]^{2}}\left|\mathbb{E} \hat{C}_{n, h}^{(.)}(u, v)-C(u, v)\right|>\varepsilon A(c)\right\}=o(1),
$$

where $0 \leq A(c) \leq 3$. 
Proposition 1 implies that

$$
\sup _{0 \leq u, v \leq 1} \frac{R_{n}}{A(c)}\left|\hat{C}_{n, \hat{h}_{n}(u, v)}^{(.)}(u, v)-\mathbb{E} \hat{C}_{n, \hat{h}_{n}(u, v)}^{(.)}(u, v)\right| \stackrel{\mathbb{P}}{\longrightarrow} 1, \quad n \rightarrow \infty,
$$

and Proposition 2 yields

$$
\sup _{0 \leq u, v \leq 1} \frac{R_{n}}{A(c)}\left|\mathbb{E} \hat{C}_{n, \hat{h}_{n}(u, v)}^{(.)}(u, v)-C(u, v)\right| \stackrel{\mathbb{P}}{\longrightarrow} 0, \quad n \rightarrow \infty .
$$

Recalling the definition of convergence in probability, we can infer from (14) that for all $\epsilon>0, \delta>0$, there exists an integer $N \in \mathbb{N}$ such that for all $n \geq N$,

$$
\mathbb{P}\left(\frac{R_{n}}{A(c)}\left|\mathbb{E} \hat{C}_{n, \hat{h}_{n}(u, v)}^{(.)}(u, v)-C(u, v)\right|>\epsilon, \forall(u, v) \in[0,1]^{2}\right)>\delta .
$$

That is

$$
\mathbb{P}\left(\mathbb{E} \hat{C}_{n, \hat{h}_{n}(u, v)}^{(.)}(u, v)-\epsilon \frac{A(c)}{R_{n}} \leq C(u, v) \leq \mathbb{E} \hat{C}_{n, \hat{h}_{n}(u, v)}^{(.)}(u, v)+\epsilon \frac{A(c)}{R_{n}}, \forall(u, v) \in[0,1]^{2}\right)>1-\delta
$$

On the other hand, we can deduce from (13) that for all $(u, v) \in[0,1]^{2}$,

$$
\mathbb{E} \hat{C}_{n, \hat{h}_{n}(u, v)}^{(.)}(u, v)=\hat{C}_{n, \hat{h}_{n}(u, v)}^{(.)}(u, v) \pm\left(1+o_{\mathbb{P}}(1)\right) \frac{A(c)}{R_{n}} .
$$

Now, we discuss two cases. First, if

$$
\mathbb{E} \hat{C}_{n, \hat{h}_{n}(u, v)}^{(.)}(u, v)=\hat{C}_{n, \hat{h}_{n}(u, v)}^{(.)}(u, v)-\left(1+o_{p}(1)\right) \frac{A(c)}{R_{n}},
$$

then (15) is equivalent to

$$
\begin{aligned}
\mathbb{P}\left(\hat{C}_{n, \hat{h}_{n}(u, v)}^{(.)}(u, v)\right. & -E_{n}(\epsilon)-o_{p}(1) \frac{A(c)}{R_{n}} \leq C(u, v) \\
& \left.\leq \hat{C}_{n, \hat{h}_{n}(u, v)}^{(.)}(u, v)-\Delta_{n}(\epsilon)-o_{p}(1) \frac{A(c)}{R_{n}}, \forall(u, v) \in[0,1]^{2}\right)>1-\delta
\end{aligned}
$$

Thus, for any $0<\tau<1$ and for all large $n$, we can write

$$
\begin{aligned}
\mathbb{P}\left(\hat{C}_{n, \hat{h}_{n}(u, v)}^{(.)}(u, v)\right. & -E_{n}(\epsilon)-\tau \frac{A(c)}{R_{n}} \leq C(u, v) \\
& \left.\leq \hat{C}_{n, \hat{h}_{n}(u, v)}^{(.)}(u, v)-\Delta_{n}(\epsilon)-\tau \frac{A(c)}{R_{n}}, \forall(u, v) \in[0,1]^{2}\right)>1-\delta
\end{aligned}
$$

Second, whenever

$$
\mathbb{E} \hat{C}_{n, \hat{h}_{n}(u, v)}^{(.)}(u, v)=\hat{C}_{n, \hat{h}_{n}(u, v)}^{(.)}(u, v)+\left(1+o_{p}(1)\right) \frac{A(c)}{R_{n}}
$$


then, as in the first case, we can infer from (15) that for any given $0<\tau<1$ and for $n$ large enough,

$$
\begin{aligned}
\mathbb{P}\left(\hat{C}_{n, \hat{h}_{n}(u, v)}^{(.)}(u, v)\right. & +\Delta_{n}(\epsilon)+\tau \frac{A(c)}{R_{n}} \leq C(u, v) \\
& \left.\leq \hat{C}_{n, \hat{h}_{n}(u, v)}^{(.)}(u, v)+E_{n}(\epsilon)+\tau \frac{A(c)}{R_{n}}, \forall(u, v) \in[0,1]^{2}\right)>1-\delta
\end{aligned}
$$

For both cases, letting $\tau$ tends to 0 in (16) and (17) yields,

$$
\mathbb{P}\left(\hat{C}_{n, \hat{h}_{n}(u, v)}^{(.)}(u, v)-E_{n}(\epsilon) \leq C(u, v) \leq \hat{C}_{n, \hat{h}_{n}(u, v)}^{(.)}(u, v)-\Delta_{n}(\epsilon), \forall(u, v) \in[0,1]^{2}\right)>1-\delta
$$

or

$$
\mathbb{P}\left(\hat{C}_{n, \hat{h}_{n}(u, v)}^{(.)}(u, v)+\Delta_{n}(\epsilon) \leq C(u, v) \leq \hat{C}_{n, \hat{h}_{n}(u, v)}^{(.)}(u, v)+E_{n}(\epsilon), \forall(u, v) \in[0,1]^{2}\right)>1-\delta .
$$

Now, by observing that

$$
\hat{C}_{n, \hat{h}_{n}(u, v)}^{(.)}(u, v)-\Delta_{n}(\epsilon) \leq \hat{C}_{n, \hat{h}_{n}(u, v)}^{(.)}(u, v)+\Delta_{n}(\epsilon),
$$

we conclude, for any $\epsilon>0, \delta>0$, that

$$
\mathbb{P}\left(C(u, v) \in\left[\hat{C}_{n, \hat{h}_{n}(u, v)}^{(.)}(u, v)-E_{n}(\epsilon), \hat{C}_{n, \hat{h}_{n}(u, v)}^{(.)}(u, v)+E_{n}(\epsilon)\right], \forall(u, v) \in[0,1]^{2}\right)>1-\delta
$$

and

$$
\mathbb{P}\left(C(u, v) \notin\left[\hat{C}_{n, \hat{h}_{n}(u, v)}^{(.)}(u, v)-\Delta_{n}(\epsilon), \hat{C}_{n, \hat{h}_{n}(u, v)}^{(.)}(u, v)+\Delta_{n}(\epsilon)\right], \forall(u, v) \in[0,1]^{2}\right)<\delta .
$$

Thus, (9) and (10) hold.

Remark. If (9) and (10) hold jointly for all $0<\epsilon<1$, then the intervals

$$
\left.A_{n}^{(\cdot)}(u, v), B_{n}^{(\cdot)}(u, v)\right]=\left[\hat{C}_{n, \hat{h}_{n}(u, v)}^{(\cdot)}(u, v)-\frac{A(c)}{R_{n}}, \hat{C}_{n, \hat{h}_{n}(u, v)}^{(\cdot)}(u, v)+\frac{A(c)}{R_{n}}\right], \quad 0 \leq u, v \leq 1,
$$

define asymptotic simultaneous confidence bands for the copula function $C(u, v)$. These bands are asymptotically optimal in terms of coverage probability, which tends to 1 , as $n \rightarrow \infty$. Taking $(\cdot)=(L L),(M R),(T)$ gives confidence bands for the copula function $C$ based on the three estimators, respectively.

One can observe that the upper tail dependence coefficient $\lambda_{U}$ may be written as

$$
\lambda_{U}=2-\lim _{u \rightarrow 1^{-}} \frac{1-C(u, u)}{1-u} .
$$

Since the copula $C(u, v)$ admits partial derivatives, then the diagonal function $\delta_{C}: u \mapsto C(u, u)$ is differentiable on $] 0,1\left[\right.$, and the quantity $\lim _{u \rightarrow 1^{-}} \frac{1-C(u, u)}{1-u}$ exists, as well as the quantity $\lim _{u \rightarrow 0^{+}} \frac{C(u, u)}{u}$, which corresponds to $\lambda_{L}$, the lower tail dependence 
coefficient.

Now, we provide confidence bounds for the coefficients $\lambda_{U}$ and $\lambda_{L}$. From (20), we can deduce, for all large $n$ and with probability tending to 1 , that

$$
\hat{A}_{n}^{(\cdot)}(u, u) \leq C(u, u) \leq \hat{B}_{n}^{(\cdot)}(u, u), \quad u \in[0,1],
$$

which yields

$$
a_{n}^{U}:=2-\lim _{u \rightarrow 1^{-}} \frac{1-\hat{A}_{n}^{(\cdot)}(u)}{1-u} \leq \lambda_{U} \leq 2-\lim _{u \rightarrow 1^{-}} \frac{1-\hat{B}_{n}^{(\cdot)}(u)}{1-u}=: b_{n}^{U}
$$

and

$$
a_{n}^{L}:=\lim _{u \rightarrow 0^{+}} \frac{\hat{A}_{n}^{(\cdot)}(u)}{u} \leq \lambda_{L} \leq \lim _{u \rightarrow 0^{+}} \frac{\hat{B}_{n}^{(\cdot)}(u)}{u}:=b_{n}^{L} .
$$

To approximate the limit terms in (21) and (22), we make use of an intuitive approach which consists of evaluating the above fractions over a finite number $m$ of points in the right-vicinity of 0 and the left-vicinity of 1 and to consider, for each fraction, the median of the obtained series as an estimate of its limit. The resulting bounds, denoted by $\left[a_{n}^{U}, b_{n}^{U}\right]$ for $\lambda_{U}$ and $\left[a_{n}^{L}, b_{n}^{L}\right]$ for $\lambda_{L}$, provide nonparametric asymptotic confidence intervals for $\lambda_{U}$ and $\lambda_{L}$, respectively.

\section{Applications}

\subsection{Simulation study}

To evaluate the performance of our confidence intervals, we make experiments with two Archimedian copula families : Clayton and Gumbel. The Clayton copula can be used to determine the lower tail dependence of extreme events using the formula $\lambda_{L}=2^{-\frac{1}{\theta}}$. While the Gumbel's copula characterizes the upper tail extreme dependence, with the expression $\lambda_{U}=2-2^{\frac{1}{\theta}}$.

For each copula $C_{\theta}$ with parameter $\theta$, we generate $n$ couples of random observations $\left(u_{i}, v_{i}\right), i=1, \cdots, n$, using the conditional sampling method and calculate the value of the three estimators defined in Section 2 by integrating the Epanechnikov kernel, $k(x)=$ $0.75\left(1-x^{2}\right) \mathbb{I}(|x| \leq 1)$. The variable bandwidth $h=\hat{h}_{n}(u, v)$ is defined as $h=h_{n} b(u, v)$ where the function $b(u, v)$ is given by (7), with $\alpha=1 / 2$. It is shown that ( see, e.g., Chen and Huang, 2007) the optimal bandwidth, while minimizing the mean square error of kernel copula estimators, is of the form $C_{0} n^{-1 / 3}$. So, we may choose the deterministic sequence of bandwidths $h_{n}$, as equal to $0.5 n^{-1 / 3}$, for suitable calculations. Finally, we compute the confidence intervals $\left[a_{n}^{U}, b_{n}^{U}\right]$ and $\left[a_{n}^{L}, b_{n}^{L}\right]$ for $\lambda_{U}$ and $\lambda_{L}$, respectively. Although the constant $A(c)$ is not arbitrary, it should be defined in such a way that the resulting bounds do not exceed 1 or are not less than 0 . Since in these simulations, we arbitrarily fix the sample size $n$, and then $R_{n}$, we must define $0<A(c)<3$ to obtain adequate bounds. A suitable value for obtaining adequate results may be $A(c)=0.16$. In computing the bounds $a_{n}^{U}, b_{n}^{U}$ or $a_{n}^{L}, b_{n}^{L}$, we consider $B=1000$ replications and report the mean values as estimates for $a_{n}^{U}, b_{n}^{U}$ or $a_{n}^{L}, b_{n}^{L}$. With a sample size of $n=100$, we obtain the results in the tables below.

Journal home page: www.jafristat.net ; www.projecteuclid.org/as 
In Table 1, we give the results for $\lambda_{L}$ in case of Clayton family. For arbitrary values for the parameter $\theta=1,3,5$, we observe that the confidence intervals contain the true value of the lower tail dependence coefficient $\lambda_{L}$, showing the performance of the bounds.

Table 2 shows the results for the Gumbel's case. Here, we also observe that our confidence intervals contain the exact value of the upper tail dependence coefficient $\lambda_{U}$, for $\theta=3,5$. But the results are not successful for $\theta=1$. We do not have the explanation for this, but we think that it may be depend on the fact that $\theta=1$ is the lower bound for the set of parameter of the Gumbel's copula.

In both cases, the experiments show that, for larger values of the parameter as $\theta=10$, the confidence intervals fail to contain the true values of $\lambda_{L}$ or $\lambda_{U}$. This might be caused by the fact that when $\theta$ is large enough, the exact values of $\lambda_{L}=2^{-1 / \theta}$ and $\lambda_{U}=2-2^{1 / \theta}$ are too close to 1 .

\begin{tabular}{cccccccccc}
\hline & \multicolumn{3}{c}{$\theta=1$} & \multicolumn{3}{c}{$\theta=3$} & \multicolumn{3}{c}{$\theta=5$} \\
\hline Estimator & $a_{n}^{L}$ & $\lambda_{L}$ & $b_{n}^{L}$ & $a_{n}^{L}$ & $\lambda_{L}$ & $b_{n}^{L}$ & $a_{n}^{L}$ & $\lambda_{L}$ & $b_{n}^{L}$ \\
\hline Local linear & 0.24 & 0.25 & 0.52 & 0.45 & 0.79 & 0.89 & 0.48 & 0.87 & 0.94 \\
Mirror-Reflection & 0.20 & 0.50 & 0.67 & 0.41 & 0.79 & 0.88 & 0.47 & 0.87 & 0.97 \\
Transformation & 0.22 & 0.50 & 0.75 & 0.07 & 0.79 & 0.93 & 0.04 & 0.87 & 0.92 \\
\hline
\end{tabular}

Table 1. Confidence intervals for $\lambda_{L}$ in case of Clayton copula.

\begin{tabular}{ccccccc}
\hline & \multicolumn{3}{c}{$\theta=3$} & \multicolumn{3}{c}{$\theta=5$} \\
\hline Estimator & $a_{n}^{U}$ & $\lambda_{U}$ & $b_{n}^{U}$ & $a_{n}^{U}$ & $\lambda_{U}$ & $b_{n}^{U}$ \\
\hline Local linear & 0.31 & 0.74 & 0.75 & 0.50 & 0.85 & 0.88 \\
Mirror-reflection & 0.48 & 0.74 & 0.85 & 0.53 & 0.85 & 0.89 \\
Transformation & 0.40 & 0.74 & 0.86 & 0.53 & 0.85 & 0.89 \\
\hline
\end{tabular}

Table 2. Confidence intervals for $\lambda_{U}$ in case of Gumbel's copula.

\subsection{Application to real data sets}

We consider data from the stock index of CAC 40 quoted by Euronext (main financial operator of Euro zone). The data consist of daily quotations for the opening price $(X)$ and the closing price $(Y)$, and are recorded during the period from 23/04/2015 to 02/01/2016. This corresponds to 200 observations of the couple $(X, Y)$. Our goal is to test for the tail dependence between the opening price and the closing price. Figure 1 exibits dependence on the upper tail of the distribution of the couple $(X, Y)$. So, we are going to test this upper tail dependence by providing confidence intervals for the coefficient $\lambda_{U}$, based on the three studied estimators. The results are summarized in the following Table 3.

Journal home page: www.jafristat.net ; www.projecteuclid.org/as 


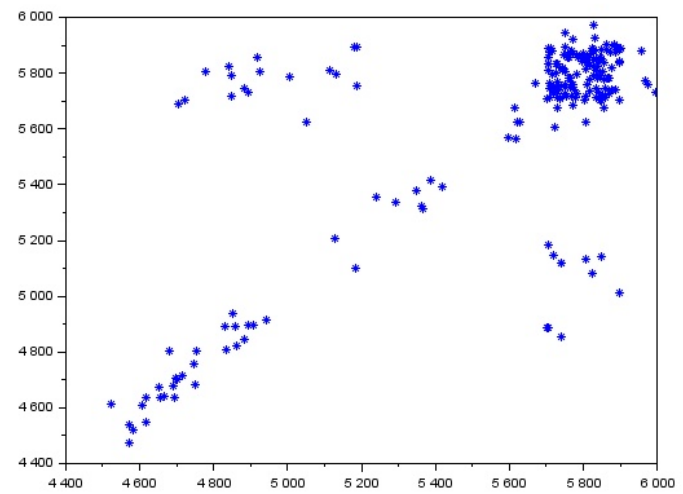

Fig. 1. Scatter plot of the observed data, size $n=200$.

\begin{tabular}{c|c|c}
\hline Estimator & $a_{n}^{U}$ & $b_{n}^{U}$ \\
\hline Local linear & 0.73 & 0.85 \\
Mirror-reflection & 0.88 & 0.99 \\
Transformation & 0.84 & 0.92 \\
\hline
\end{tabular}

Table 3. Estimation of $\lambda_{U}$ by confidence intervals

We observe that the coefficient $\lambda_{U}$ is strictly different from 0 , for all the three estimators. Thus, we can say that $X$ and $Y$ are asymptotically dependent on the upper tail level of their joint distribution. This means that, there is an extremal dependence between large values of opening prices and closing prices in the considered period.

\section{Appendix}

\section{Proof of Proposition 1.}

It results from a combination of a general theorem of Mason and Swanepoel (2010) for proving the uniform in bandwidth consistency of kernel-type function estimators, and a law of the iterated logarithm for Kiefer processes due to Wichura (1973).

Let $\phi:[0,1] \mapsto[0,1]$ be an increasing transformation. For a bivariate kernel $K(\cdot, \cdot)$ and $0<u, v<1$, define the estimator

$$
\hat{C}_{n, h}^{(\cdot)}(u, v)=\frac{1}{n} \sum_{i=1}^{n} K\left(\frac{\phi^{-1}(u)-\phi^{-1}\left(\hat{U}_{i}\right)}{h}, \frac{\phi^{-1}(v)-\phi^{-1}\left(\hat{V}_{i}\right)}{h}\right)
$$

and set

$$
\hat{D}_{n, h}^{(\cdot)}(u, v)=: \hat{C}_{n, h}^{(\cdot)}(u, v)-\mathbb{E} \hat{C}_{n, h}^{(\cdot)}(u, v)
$$


We call $\hat{D}_{n, h}^{(\cdot)}(u, v)$ the deviation of the estimator $\hat{C}_{n, h}^{(\cdot)}(u, v)$ from its expectation and we will study its behavior by using general empirical process theory.

We remark that if the kernel $K(\cdot, \cdot)$ is multiplicative, i.e. $K(x, y)=K(x) K(y)$, we obtain directly the transformation estimator (6). If we set $\phi(t)=t$, the identity function and employ the local linear kernels $K_{u, h}(\cdot)$ and $K_{v, h}(\cdot)$, then we obtain the local linear kernel estimator (4). For the mirror-reflection estimator (5), we consider the following decomposition due to Omelka et al. (2009),

$$
\hat{C}_{n}^{(M R)}(u, v)=\sum_{l=1}^{9}\left[Z_{n}(l, u, v)-Z_{n}(l, u, 0)-Z_{n}(l, 0, v)+Z_{n}(l, 0,0)\right],
$$

where

$$
Z_{n}(l, u, v)=\frac{1}{n} \sum_{i=1}^{n} K\left(\frac{u-\hat{U}_{i}^{(l)}}{h_{n}}\right) K\left(\frac{v-\hat{V}_{i}^{(l)}}{h_{n}}\right) .
$$

Setting $\phi(t)=t$ and using a multiplicative kernel, one can see that each quantity $Z_{n}(l, u, v)$ may be put in the form (23).

Now, let $H_{n}, F_{n}$ and $G_{n}$ be the empirical cumulative distribution functions of $H, F$ and $G$, respectively. Then the estimator based directly on Sklar's Theorem is given by

$$
C_{n}(u, v)=H_{n}\left(F_{n}^{-1}(u), G_{n}^{-1}(v)\right),
$$

with $F_{n}^{-1}(u)=\inf \left\{x: F_{n}(x) \geq u\right\}$ and $G_{n}^{-1}(v)=\inf \left\{x: F_{n}(x) \geq v\right\}$. The bivariate empirical copula process is defined as

$$
\mathbb{C}_{n}(u, v)=\sqrt{n}\left[C_{n}(u, v)-C(u, v)\right], \quad(u, v) \in[0,1]^{2} .
$$

Introduce the following quantity.

$$
\widetilde{C}_{n}(u, v)=\frac{1}{n} \sum_{i=1}^{n} \mathbb{I}\left\{U_{i} \leq u, V_{i} \leq v\right\}
$$

which represents the uniform bivariate empirical distribution function based on a sample $\left(U_{1}, V_{1}\right), \cdots,\left(U_{n}, V_{n}\right)$ of i.i.d random variables uniformly distributed on $[0,1]^{2}$. Define the following empirical process

$$
\widetilde{\mathbb{C}}_{n}(u, v)=\sqrt{n}\left[\widetilde{C}_{n}(u, v)-C(u, v)\right], \quad(u, v) \in[0,1]^{2} .
$$

Then, we can easily prove that that

$$
\widetilde{\mathbb{C}}_{n}(u, v)=\mathbb{C}_{n}(u, v)+\frac{1}{\sqrt{n}} .
$$

For any given increasing transformation $\phi$ and $(u, v) \in[0,1]^{2}$, define

$$
\begin{gathered}
g_{n, h}=\hat{C}_{n, h}^{(\cdot)}(u, v)-\widetilde{C}_{n}(u, v) \\
=\frac{1}{n} \sum_{i=1}^{n}\left[K\left(\frac{\phi^{-1}(u)-\phi^{-1}\left(\hat{U}_{i}\right)}{h}, \frac{\phi^{-1}(v)-\phi^{-1}\left(\hat{V}_{i}\right)}{h}\right)-\mathbb{I}\left\{U_{i} \leq u, V_{i} \leq v\right\}\right] \\
=\frac{1}{n} \sum_{i=1}^{n}\left[K\left(\frac{\phi^{-1}(u)-\phi^{-1}\left(\hat{F}_{n} o F^{-1}\left(U_{i}\right)\right)}{h}, \frac{\phi^{-1}(v)-\phi^{-1}\left(\hat{G}_{n} o G^{-1}\left(V_{i}\right)\right)}{h}\right)-\mathbb{I}\left\{U_{i} \leq u, V_{i} \leq v\right\}\right] \\
=: \frac{1}{n} \sum_{i=1}^{n} g\left(U_{i}, V_{i}, h\right),
\end{gathered}
$$

Journal home page: www.jafristat.net ; www.projecteuclid.org/as 
where $g$ belongs to the class of measurable functions $\mathcal{G}$ defined as

$$
\mathcal{G}=\left\{\begin{array}{c}
g:(s, t, h) \mapsto g(s, t, h)=K\left(\frac{\phi^{-1}(u)-\phi^{-1}\left(\zeta_{1, n}(s)\right)}{h}, \frac{\phi^{-1}(v)-\phi^{-1}\left(\zeta_{2, n}(t)\right)}{h}\right)-\mathbb{I}\{s \leq u, t \leq v\}, \\
u, v \in[0,1], 0<h<1 \text { and } \zeta_{1, n} \zeta_{2, n}:[0,1] \mapsto[0,1] \text { nondecreasing. }
\end{array}\right\}
$$

Since $\mathbb{E} \widetilde{C}_{n}(u, v)=C(u, v)$, one can observe that

$$
\sqrt{n}\left|g_{n, h}-\mathbb{E} g_{n, h}\right|=\left|\sqrt{n} \hat{D}_{n, h}^{(\cdot)}(u, v)-\widetilde{\mathbb{C}}_{n}(u, v)\right| .
$$

Introduce the following conditions on the class of functions $\mathcal{G}$.

(G.i) There exists a finite constant $\kappa>0$ such that

$$
\sup _{0 \leq h \leq 1} \sup _{g \in \mathcal{G}}\|g(\cdot, \cdot, h)\|_{\infty}=\kappa<\infty .
$$

(G.ii) There exists a constant $C^{\prime}>0$ such that for all $h \in[0,1]$,

$$
\sup _{g \in \mathcal{G}} \mathbb{E}\left[g^{2}(U, V, h)\right] \leq C^{\prime} h \text {. }
$$

(F.i) $\mathcal{G}$ satisfies the uniform entropy condition, i.e.,

$$
\exists C_{0}>0, \nu_{0}>0: N(\epsilon, \mathcal{G}) \leq C_{0} \epsilon^{-\nu_{0}} .
$$

(F.ii) $\mathcal{G}$ is a pointwise measurable class, i.e there exists a countable sub-class $\mathcal{G}_{0}$ of $\mathcal{G}$ such that for all $g \in \mathcal{G}$, there exits $\left(g_{m}\right)_{m} \subset \mathcal{G}_{0}$ such that $g_{m} \longrightarrow g$.

Proposition 3. Suppose that the copula function $C$ has bounded first order partial derivatives on $(0,1)^{2}$ and the transformation $\phi$ admits a bounded derivative $\phi^{\prime}$. Then assuming (G.i), (G.ii), (F.i) and (F.ii), we have for some $c>0,0<h_{0}<1$, with probability one,

$$
\limsup _{n \rightarrow \infty} \sup _{\frac{c \log n}{n} \leq h \leq h_{0}} \sup _{(u, v) \in(0,1)^{2}} \frac{\left|\sqrt{n} \hat{D}_{n, h}^{(\cdot)}(u, v)-\tilde{\mathbb{C}}_{n}(u, v)\right|}{\sqrt{h(|\log h| \vee \log \log n)}}=A(c),
$$

where $A(c)$ is a positive constant.

Corollary 1. Under the assumptions of Proposition 3, one has for any sequence of constants $0<b_{n}<1$, satisfying $b_{n} \rightarrow 0, b_{n} \geq(\log n)^{-1}$, with probability one,

$$
\sup _{\frac{c \log n}{n} \leq h \leq b_{n}} \sup _{(u, v) \in(0,1)^{2}} \frac{\left|\sqrt{n} \hat{D}_{n, h}^{(\cdot)}(u, v)-\tilde{\mathbb{C}}_{n}(u, v)\right|}{\sqrt{\log \log n}}=O\left(\sqrt{b_{n}}\right) .
$$

To avoid making the paper any longer again, we refer to Ba et al. (2015), Theorem 1, for the proof of this Proposition 3 and its Corollary 1. Coming back to the proof of Proposition 1 , we have to show that

$$
\limsup _{n \rightarrow \infty} \sup _{\frac{c \log n}{n} \leq h \leq b_{n}} \sup _{(u, v) \in[0,1]^{2}} \frac{\left|\sqrt{n} \hat{D}_{n, h}^{(\cdot)}(u, v)\right|}{\sqrt{2 \log \log n}} \leq 3 .
$$


Towards this end, we make use of an approximation of the empirical copula process $\mathbb{C}_{n}$ by a Kiefer process (see e.g., Zari, 2010, page 100). Let $\mathbb{W}(u, v, t)$ be a 3 -parameters Wiener process defined on $[0,1]^{2} \times[0, \infty)$. Then the Gaussian process $\mathbb{K}(u, v, t)=\mathbb{W}(u, v, t)-$ $\mathbb{W}(1,1, t) . u v$ is called a 3 -parameters Kiefer process defined on $[0,1]^{2} \times[0, \infty)$.

By Theorem 3.2 of Zari (2010), for $d=2$, there exists a sequence of Gaussian processes $\left\{\mathbb{K}_{C}(u, v, n), u, v \in[0,1], n>0\right\}$ such that

$$
\sup _{(u, v) \in[0,1]^{2}}\left|\sqrt{n} \mathbb{C}_{n}(u, v)-\mathbb{K}_{C}^{*}(u, v, n)\right|=O\left(n^{3 / 8}(\log n)^{3 / 2}\right),
$$

where

$$
\mathbb{K}_{C}^{*}(u, v, n)=\mathbb{K}_{C}(u, v, n)-\mathbb{K}_{C}(u, 1, n) \frac{\partial C(u, v)}{\partial u}-\mathbb{K}_{\mathbb{C}}(1, v, n) \frac{\partial C(u, v)}{\partial v} .
$$

This yields

$$
\limsup _{n \rightarrow \infty} \sup _{(u, v) \in[0,1]^{2}} \frac{\left|\mathbb{C}_{n}(u, v)\right|}{\sqrt{2 \log \log n}}=\limsup _{n \rightarrow \infty} \sup _{(u, v) \in[0,1]^{2}} \frac{\left|\mathbb{K}_{C}^{*}(u, v, n)\right|}{\sqrt{2 n \log \log n}} .
$$

By the works of Wichura (1973) on the law of the iterated logarithm , for $d=2$, one has almost surely

$$
\limsup _{n \rightarrow \infty} \sup _{(u, v) \in[0,1]^{2}} \frac{\left|\mathbb{K}_{\mathbb{C}}^{*}(u, v, n)\right|}{\sqrt{2 n \log \log n}} \leq 3
$$

which entails

$$
\limsup _{n \rightarrow \infty} \sup _{(u, v) \in[0,1]^{2}} \frac{\left|\mathbb{C}_{n}(u, v)\right|}{\sqrt{2 \log \log n}} \leq 3 .
$$

Since $\mathbb{C}_{n}(u, v)$ and $\tilde{\mathbb{C}}_{n}(u, v)$ are asymptotically equivalent in view of $(26)$, one obtains

$$
\limsup _{n \rightarrow \infty} \sup _{(u, v) \in[0,1]^{2}} \frac{\left|\tilde{\mathbb{C}}_{n}(u, v)\right|}{\sqrt{2 \log \log n}} \leq 3 .
$$

Applying Corollary 1 and the fact that $\sqrt{b_{n}} \rightarrow 0$, we obtain Proposition 1 by taking the equality to a constant $A(c)$, with $0<A(c) \leq 3$.

\section{Proof of Proposition 2.}

First, observe that for all $(u, v) \in[0,1]^{2}$,

$$
C(u, v)=\int_{-1}^{1} \int_{-1}^{1} C(u, v) k(s) k(t) d s d t
$$

where $k(\cdot)$ is the Lebesgue-derivative of a function $K(x)=\int_{-\infty}^{x} k(s) d s$.

Next, for a multiplicative kernel $K(x, y)=K(x) K(y)$, we have

$$
\mathbb{E} \hat{C}_{n, h}^{(\cdot)}(u, v)=\mathbb{E}\left[K\left(\frac{\phi^{-1}(u)-\phi^{-1}\left(\zeta_{1, n}\left(U_{i}\right)\right)}{h}\right) K\left(\frac{\phi^{-1}(v)-\phi^{-1}\left(\zeta_{2, n}\left(V_{i}\right)\right)}{h}\right)\right]
$$

Journal home page: www.jafristat.net ; www.projecteuclid.org/as 
where $\zeta_{1, n}\left(U_{i}\right)=\hat{F}_{n} \circ F^{-1}\left(U_{i}\right)=\hat{U}_{i} \quad$ and $\zeta_{2, n}\left(V_{i}\right)=\hat{F}_{n} \circ F^{-1}\left(V_{i}\right)=\hat{V}_{i}$. One can easily prove that

$$
\mathbb{E} \hat{C}_{n, h}^{(\cdot)}(u, v)=\int_{-1}^{1} \int_{-1}^{1} C\left(\zeta_{1, n}^{-1} \circ \phi\left(\phi^{-1}(u)-s h\right), \zeta_{2, n}^{-1} \circ \phi\left(\phi^{-1}(v)-t h\right)\right) k(s) k(t) d s d t .
$$

So, the bias term

$$
\begin{aligned}
B_{n, h}^{(\cdot)}(u, v) & =\mathbb{E} \hat{C}_{n, h}^{(\cdot)}(u, v)-C(u, v) \\
& =\int_{-1}^{1} \int_{-1}^{1}\left[C\left(\zeta_{1, n}^{-1} \circ \phi\left(\phi^{-1}(u)-s h\right), \zeta_{2, n}^{-1} \circ \phi\left(\phi^{-1}(v)-t h\right)\right)-C(u, v)\right] k(s) k(t) d s d t .
\end{aligned}
$$

The empirical kernel distributions $\hat{F}_{n}$ and $\hat{G}_{n}$ are assumed to be asymptotically equivalent to the classical empirical distribution functions $F_{n}$ and $G_{n}$. Thus, from the Chung (1949) law of the iterated logarithm, we can infer that for all $u \in[0,1]$, as $n \rightarrow \infty$,

$$
\zeta_{1, n}^{-1}(u)-u=F \circ \hat{F}_{n}^{-1}(u)-F \circ F^{-1}(u)=O\left(n^{-1} \log \log n\right) .
$$

That is, $\zeta_{1, n}^{-1}(u)$ is asymptotically equivalent to $u$. Same for $\zeta_{2, n}^{-1}(u)=G \circ \hat{G}_{n}^{-1}(u)$. Hence, for large enough $n$, we have

$$
B_{n, h}^{(\cdot)}(u, v)=\int_{-1}^{1} \int_{-1}^{1}\left[C\left(\phi\left(\phi^{-1}(u)-s h\right), \phi\left(\phi^{-1}(v)-t h\right)\right)-C(u, v)\right] k(s) k(t) d s d t .
$$

By a Taylor expansion of 2-order, and taking account of the symmetry of the function $k(\cdot)$, which implies that

$$
\int_{-1}^{1} s k(s) d s=0
$$

we get for all $(u, v) \in[0,1]^{2}$ and large enough $n$,

$$
\begin{aligned}
B_{n, h}^{(\cdot)}(u, v) & =h^{2} \int_{-1}^{1} \int_{-1}^{1}\left[s^{2} \frac{\partial^{2} C(u, v)}{\partial u^{2}}+s t \frac{\partial^{2} C(u, v)}{\partial u \partial v}+s^{2} \frac{\partial^{2} C(u, v)}{\partial v^{2}}\right] k(s) k(t) d s d t \\
& =h^{2}\left[\int_{-1}^{1} s^{2} \frac{\partial^{2} C(u, v)}{\partial u^{2}} k(s) d s+\int_{-1}^{1} t^{2} \frac{\partial^{2} C(u, v)}{\partial v^{2}} k(t) d t\right] .
\end{aligned}
$$

From this and the boundedness of the second-order derivatives of the copula $C$, we conclude that

$$
\sup _{\frac{c \log n}{n} \leq h \leq b_{n}} \sup _{(u, v) \in[0,1]^{2}} B_{n, h}^{(T)}(u, v)=O\left(b_{n}^{2}\right) .
$$

and

$$
\left(\frac{n}{2 \log \log n}\right)^{1 / 2} \sup _{\frac{c \log n}{n} \leq h \leq b_{n}} \sup _{(u, v) \in[0,1]^{2}} B_{n, h}^{(T)}(u, v)=O\left(\frac{\sqrt{n} b_{n}^{2}}{\sqrt{2 \log \log n}}\right)=o(1) .
$$

\section{Acknowledgements}

The authors are very grateful to the referees for their careful reading of this paper and for their constructive criticism that result in an improved version. 


\section{References}

Ba, D., Seck, C.T. and Lo, G.S., 2015. Asymptotic Confidence Bands for Copulas Based on the Local Linear Kernel Estimator. Applied Mathematics, 6, 2077-2095.

Chen, S.X. and Huang, T.-M., 2007. Nonparametric estimation of copula functions for dependence modeling. Canadian Journal of Statistics, 35, 265-282.

Chung, K-L, 1949. An estimate concerning the kolmogoroff limit distribution. Trans Am.Math Soc, 67, 36-50.

Coles, S.G., Heffernan, J., and Tawn, J.A., 1999. Dependence measures for extreme value analyses. Extremes, 3, 5-38.

Deheuvels, P. and Mason, D. M., 2004. General Asymptotic Confidence Bands Based on Kernel-type Function Estimators. Statistical Inference stochastic process, 7, 225-277.

Deheuvels, P., 1979. La fonction de dépendence empirique et ses propriétés. Un test non paramétrique d'indépendance. Bulletin Royal Belge de l'Académie des Sciences, 65, 274292.

Fermanian, J.D. and Scaillet, O., 2003. Nonparametric Estimation of Copulas for Time Series. Journal of risk, 5, 25-54.

Fermanian, J., Radulovic, D. and Wegkamp, M., 2004. Weak convergence of empirical copula processes. International Statistical Institute (ISI) and Bernoulli Society for Mathematical Statistics and Probability, 10, 847-860.

Genest, C., Ghoudi, K. and Rivest, L.P., 1995. A semiparametric estimation procedure of dependence parameters in multivariate families of distributions. Biometrika, 82, 543-552.

Gijbels, I. and Mielniczuk, J., 1990. Estimation of the density of a copula function. Comm. Statist. Theory Methods 19, 445-464.

Joe, H., 1997. Multivariate Models and Dependence Concepts, Chapman and Hall. London.

Juri, A., Wüthrich, M.V., 2002. Copula convergence theorems for tail events. Insurance : Mathematics and Economics, 24, 139-148.

Ledford, A. and Tawn, J.A., 1998. Concomitant tail behaviour for extremes. Advances in applied Probability, 30, 197-215.

Malevergne, Y. and Sornette, D., 2004. How to account for extreme co-movements between individual stock and the market? Journal of Risk, 6(30), 71-116.

Mason, D.M. and Swanepoel, J.H.W, 2010. A general result on the uniform in bandwidth consistency of kernel-type function estimators. TEST, 20, 72-94.

Nelsen, R.B., 1999. An Introduction to Copulas. Springer Series in Statistics.

Omelka, M. and Gijbels, I. and Veraverbeke, N., 2009. Improved kernel estimators of copulas : weak convergence and goodness-of-fit testing. The Annals of Statistics, 37, 3023-3058.

Oakes, D., 1982. A model for association in bivariate survival data. Journal of the Royal Statistical Society Series B, 44, 414-422.

Poon, S.H., Rockinger, M. and Tawn, J., 2004. Extreme-value dependence in financial markets : Diagnostics, models and financial implications. Review of Financial Studies 17, 581-610.

Sklar, A., 1959. Fonctions de répartition à $n$ dimensions et leurs marges. Publ. Inst. Statistic. Univ. Paris, 8, 229-231.

van der Vaart, A.W. and Wellner, J. A., 1996. Weak Convergence and Empirical Processes. Springer, New York. 
C.T. Seck, D. Ba and G.S. Lo, Afrika Statistika, Vol. 11(2), 2016, 1023-1039. Nonparametric confidence intervals for tail dependence based on copulas.

Schmidt, R., Stadtmüller, U., 2006. Non parametric estimation of tail dependence. The Scandinavian Journal of Statistics, 33, 307-335.

Wichura, M.J., 1973. Some Strassen-type laws of the iterated logarithm for multiparameter stochastic processes with independent increments. The Annals of Probability, 1, 272-296.

Zari, T., 2010. Contribution à l'étude du processus empirique de copule. Thèse de doctorat, Université Paris 6, France. 